\title{
MeTAPHYSIK IN DER ZEITGENÖSSISCHEN HeGEL-REZEPTION
}

In meinem Aufsatz werde ich zunächst (1.) das Programm einer »nichtmetaphysischen « Hegeldeutung rekonstruieren, die insbesondere von Pippin und Pinkard in den 90er Jahren entwickelt worden ist, dann (2.) die Auffassung des Geistes, die aus diesem Programm resultiert am Beispiel von Terry Pinkards Geistkapitel in German Philosophy. 1760-1860. The Legacy of Idealism ${ }^{1}$ erörtern. Abschließend (3.) werde ich auf die Gegentendenz zur antimetaphysischen Deutung in der zeitgenössischen Hegel-Rezeption hinweisen und zeigen, dass sowohl die Autoren (wie Pippin und Pinkard), die versuchen, die Metaphysik aus der Hegelschen Philosophie zu beseitigen, als auch diejenigen (vgl. z. B. Beiser), die auf die zentrale Rolle der Metaphysik in der Philosophie Hegels hinweisen, einige Missverständnisse bezüglich des Metaphysik- und Geistbegriffs teilen.

\section{Nichtmetaphysische Hegel-Deutungen}

In Hegel, Scepticism and Transcendental Arguments hat Robert Stern die Schwierigkeiten der kanonischen angloamerikanischen Hegel Interpretation auf die Tatsache zurückgeführt, dass Hegels Position entweder als »extremen spiritualistischen Idealismus«, demzufolge es keine »mind independent world « gibt, oder als extremen Rationalismus, demzufolge »the world is guaranteed to conform to thought as the embodiment of a quasi-Platonic conceptual structure « gedeutet worden ist. ${ }^{2} \mathrm{Er}$ behauptete ferner, dass diese Schwierigkeiten von der neueren amerikanischen Hegel Interpretation (von Charles Taylor, Robert Pippin, Terry Pinkard, u. a.), zu der er auch sich selbst zählt, beseitigt worden sind. Diese Autoren versuchen, so Stern, Hegel zu kantianisieren, um ihm »die schwere Waffe der Metaphysik zu nehmen und ihm die leichtere des Transzendentalismus zu reichen «. ${ }^{3}$

Meiner Ansicht nach teilen die alte und die neuere angloamerikanische Hegel Deutung ein Missverständnis bezüglich des spezifisch Hegelschen Metaphysikbegriffs. Im Folgenden werde ich mich auf die Rekonstruktion der Letzteren konzentrieren. Sie kann als der Versuch interpretiert werden, die frühere, platonisierende Deutung Hegels zu korrigieren.

In Hegel's Idealism: The Satisfactions of Self-Counsciousness, ${ }^{4}$ hat Robert Pippin zum ersten Mal das Programm einer "nicht metaphysischen « Hegel Interpretation vorgestellt, und betont, dass Hegel »antimetaphysisch« zu interpretieren impliziert, die Kontinuität zwischen der Hegelschen und der Kantischen und Fichteschen Philosophie zu betonen. Pippin äußert in diesem Buch das Vorhaben, den Kantischen Ursprung von Hegels Philosophie vor Augen zu stellen, der seiner Ansicht nach in dem Idealismus und in der Frage nach der transzendentalen Apperzeption aufzuspüren ist.

1 Terry Pinkard, German Philosophy. 1760-1860. The Legacy of Idealism, Cambridge, 2002.

2 Robert Stern, »Hegel, Scepticism and Transcendental Arguments«, in: Skeptizismus und spekulatives Denken in der Philosophie Hegels, hg. v. H. F. Fulda, R.-P. Horstmann, Stuttgart, 1996, 206-225.

3 Ebd.

4 Pippin, Hegel's Idealism: The Satisfactions of Self-Counsciousness, Cambridge, 1989. 
Das impliziert laut Pippin, dass Hegels Philosophie nicht metaphysisch ist, sie hat nicht mit einer Realität zu tun, die unabhängig vom Denken existiert, sondern mit: »thought's attempt to determine a priori what can be a possible thought of anything at all.$^{5}$ Daher interpretiert Pippin den Idealismus als eine Theorie, die die Metaphysik prinzipiell ausschließt. Gegen diese Auffassung kann man erstens einwenden, dass wenn man unter »Idealismus« die Konzeption versteht, derzufolge die Realität vom Denken produziert wird, dann stellt »Idealismus « in der Tat eine Konzeption der Realität, und daher eine Metaphysik dar. Zweitens, wenn »Idealismus« die Theorie bezeichnet, derzufolge es keine externe Realität gibt, kann man argumentieren dass der Idealismus - so aufgefasst - eine Theorie über die Art und Weise ist, wie die Dinge sind, und daher eine Metaphysik. Letztens, auch wenn man die Neu-Kantianische Interpretation akzeptiert, derzufolge die kritische Philosophie (im Kantischen Sinne) strukturell antimetaphysisch ist, und man annimmt, dass Hegel in diesem antimetaphysischen Sinne ein kritischer Philosoph ist, scheitert das Argument.

Kant selbst hatte nämlich die kritische Philosophie mit der Frage nach der Begründung der Metaphysik verknüpft, und zwar nicht nur in der Kritik der reinen Vernunft und in den Prolegomena, sondern auch in der berühmten vorkritischen und anti-metaphysischen Schrift »Die Träume eines Geistersehers ...«, in der er die dogmatische Metaphysik angriff, zugleich aber die Notwendigkeit betonte, eine kritische Metaphysik zu entwerfen. ${ }^{6}$ Hegels Philosophie knüpft an dieses Programm an. Hegel entwickelte nämlich eine objektive Form des Idealismus, den er als das Erbe der Metaphysik auffasste. So scheinen sich Metaphysik und Idealismus nicht auszuschließen, im Gegenteil: Hegels Philosophie und seine Theorie des objektiven Geistes sind vielmehr als Vermittlung dieser beiden Perspektiven (Idealismus und Metaphysik) interpretiert worden.?

Insgesamt bestätigt Pippins Interpretation Sterns Diagnose über die neuere angloamerikanische Hegel-Deutung, die ich oben erwähnt habe. Pippin versucht, die Metaphysik dadurch zu beseitigen, dass er auf die Kantischen Wurzeln und auf die Kantischen Themen der Hegelschen Philosophie hinweist. Aber eine solche antimetaphysische Deutung der Kantischen Philosophie ist - wie ich schon angedeutet habe - irreführend. Wenn man Kants Verwendung des Terminus »transzendental« berücksichtigt, tritt in den Vordergrund, dass Kant ihn zwar in einer neuen Bedeutung gebraucht hat, die sich von der alten, metaphysischen unterscheidet, aber auch, dass Kant (wie vor allem aus den Vorlesungen und Reflexionen ersichtlich wird) seine Transzendentalphilosophie nicht in antimetaphysischen Sinn verstand. ${ }^{8}$ Viele Autoren (die ersten waren Martin Heidegger und Gottfried Martin) haben betont, dass Kants epistemologische Wende eine neue Ontologie und Metaphysik impliziert. ${ }^{9}$

Terry Pinkards Hegel-Deutung ${ }^{10}$ kann auch zu denjenigen Interpretationen gezählt werden, die versuchen, die Rolle der Metaphysik in Hegels Philosophie zu reduzieren, indem sie deren Kanti-

5 Pippin, Hegel's Idealism, a. a. O., 184.

6 Immanuel Kant, Träume eines Geistersehers, erläutert durch die Träume der Metaphysik 1766, in: Gesammelte Werke, hg. v. der (Königlichen) Preußischen (später Deutschen) Akademie der Wissenschaften, Bd. I ff., Berlin, 1900 ff., II, 119.

7 Zur Kontinuität zwischen Hegels Theorie des objektiven Geistes und der Tradition der Ontologie und Metaphysik vgl. H.-G. Gadamer, »Die Idee der Hegelschen Logik«, in: Ders., Hegel-Husserl-Heidegger, Tübingen, 1987, 65-86.

$8 \mathrm{Vgl}$. Norbert Hinske, »Die historischen Vorlagen der Kantischen Transzendentalphilosophie«, in: Archiv für Begriffsgeschichte 12 (1968), 86-113; ders: Kants Weg zur Transzendentalphilosophie, Stuttgart, 1970; ders: Stichwortartikel »Transzendental; Transzendentalphilosophie« in: Historisches Wörterbuch der Philosophie, hg. v. J. Ritter und K. Gründer, Darmstadt, 1998, 1376-1388.

9 Vgl. Martin Heidegger, Kant und das Problem der Metaphysik, Frankfurt/M., $1998^{6}$.

10 Pinkard, Hegel's Phenomenology. The Sociality of Reason, Cambridge MA, 1996 und ders.: "What is the Non Metaphysical Reading of Hegel? A Reply to Frederick Beiser«, in: Bulletin of the Hegel Society of Great Britain, 34 (1996), 13-20. 
sche Voraussetzungen aufdecken. Aber anders als Pippin, betont Pinkard, der Interpretation seines Lehrers Klaus Hartmann zu folgen, derzufolge Hegels Philosophie (und insbesondere Hegels Logik) als Ontologie (im Sinne einer Kategorienlehre) verstanden werden kann. Pinkard - in einem Sprachgebrauch, der auf den späten Heidegger zurückgeht - geht daher von der Annahme einer radikalen Differenz zwischen den beiden Termini »Metaphysik« und »Ontologie« aus. Diese Trennung lässt sich aber weder durch die Hegelschen Texte noch durch die Auseinandersetzung mit der Geschichte des Begriffs »Metaphysik« belegen. Wie Tom Rockmore in Hegel's Metaphysics, or the Categorial Approach to Knowledge of Experience hervorgehoben hat: "Hartmann is correct about Hegel, but, unfortunately, (...) he is non-Hegelian in his choice of terminology. What Hartmann calls snon-metaphysical ' should be called >metaphysical s since Hegel's hermeneutic of categories is in fact (...) a metaphysical theory «. ${ }^{11}$ Wenn man die Geschichte des Terminus »Metaphysik« und seine Semantik in dem Hegelschen Text berücksichtigt, kommt zum Vorschein, dass Ontologie als Theorie der Kategorien und Metaphysik nicht entgegengesetzt sind. Wie Rockmore hervorhebt: "Since Aristotle, the term >metaphysics has frequently been taken to mean first philosophy, especially ontology «. ${ }^{12}$

Daher ist der Versuch der neueren angloamerikanischen Deutungen, auf die Kontinuität zwischen Kant und Hegel hinzuweisen begründet. Gegen Pippins Versuch in Hegel's Idealism, auf die transzendentale Apperzeption als das Grundthema des Deutschen Idealismus und als das Element der Kontinuität zwischen Kantischer, Fichtescher und Hegelscher Philosophie hinzuweisen, ist nichts einzuwenden; ebenfalls nicht gegen Pinkards und Hartmanns Interpretation der Hegelschen Philosophie als Hermeneutik der Kategorien. Das, was dabei problematisch ist, ist die Bezeichnung solcher Theorien als »nicht metaphysisch«.

Im Folgenden werde ich mich auf die Konsequenzen und Implikationen der »nicht metaphysischen« Deutung Hegels für die Interpretation des Begriffs des Geistes konzentrieren. Ich werde dabei Pinkards Deutung des Geistbegriffs in German Philosophy. 1760-1860. The Legacy of Idealism, ${ }^{13}$ berücksichtigen.

\section{Nicht-metaphysische Deutung des Geistbegriffs}

In German Philosophy. 1760-1860. The Legacy of Idealism, spricht Pinkard in einem Kapitel zu Hegels Geistbegriff über den Übergang von der Natur zum Geist in Hegels Enzyklopädie. Die Naturwissenschaft erfüllt hier die Funktion - so Pinkard - eines Schritts zur Erlangung des eigentlichen Ziels des Menschen, nämlich Freiheit. Der Übergang zum Geist ist dadurch gekennzeichnet, dass der Mensch versteht, dass die Differenz zwischen Natur und Geist ein Produkt des Geistes und durch den Geist selbst gesetzt ist. Das bedeutet in Pinkards Interpretation, dass diese Differenz "normativ« und nicht "metaphysisch « ist. ${ }^{14}$ Sie ist »a social achievement about what is appropriate and not appropriate to do with respect to >purely` natural creatures and the >minded c creatures we are «. ${ }^{15}$

Laut Pinkard ist Hegels Konzeption des Verhältnisses von Natur und Geist durch die Kritik an dem Kantischen Dualismus von Natur und Freiheit motiviert, ein Dualismus, den er als das »Kantische Dilemma« bezeichnet. Um Natur und Freiheit zu versöhnen, hatte Kant eine transzendentale Freiheit postuliert, die nicht den natürlichen Gesetzen unterworfen ist. Andere postkantische Philo-

11 Tom Rockmore, »Hegel's Metaphysics, or the Categorial Approach to Knowledge of Experience«, in: Hegel Reconsidered. Beyond Metaphysics and the Authoritarian State, hg. v. H. T. Engelhardt und T. Pinkard, 45.

12 Tom Rockmore, »Hegel's Metaphysics«, a. a. O., 45.

13 Pinkard, German Philosophy. 1760-1860. The Legacy of Idealism, Cambridge, 2002.

14 Pinkard, German Philosophy, a. a. O., 276.

15 Ebd. 
sophen hatten versucht, das Dualismus Dilemma dadurch aufzulösen, dass sie auf die ästhetische Erfahrung als eine Form der Erkenntnis hingewiesen hatten, die weder Natur noch Freiheit ist und somit eine Art übersinnlichen Grund der Erscheinungen ausmacht. Hegels Weg aus dem Dilemma ist dagegen laut Pinkard, "generalizing the Kantian paradox into a thesis about normative authority in general $\ll{ }^{16}$ D. h. Kants Theorie über Natur und Geist werde von Hegel verallgemeinert und als These über normative Autorität interpretiert. Hegel interpretiere den Unterschied zwischen Natur und Geist nicht als Differenz zwischen zwei getrennten »Reichen«, das eine empirisch, das andere transzendental, sondern - so Pinkard - normativ, als Frage nach der Wahrheit und nach den Gesetzen, die ins Spiel gebracht werden müssen, um diese Trennung aufzuheben. "It is thus a normative issue, not a matter of metaphysics in the sense that it is definitely not a matter of whether nature is extended matter and spirit is non-extended mental substance «. ${ }^{17}$

Pinkard fügt hinzu $» \mathrm{Or}$, as we have already put it, the distinction between nature and spirit is itself a s spiritual, ( that is, normative distinction >posited ‘ by spirit itself « ${ }^{18}$ Daher habe der Ausdruck »Geist « bei Hegel, den Pinkard durch »mindedness « übersetzt, eine besondere, nicht naturalistische, nicht dualistische Bedeutung und bezeichne darüber hinaus nicht eine der Trennung von Natur und Geist vorausgehende übersinnliche Substanz, sondern er weise vielmehr auf eine normative Instanz hin und impliziere »assuming certain responsibilities in a social space - of undertaking committments, attributing entitlements, and negotiating, as it were, the entire set of normative responses to all those related activities $\ll .{ }^{19}$

Für Pinkard ist nicht nur der Geist eine normative Instanz, sondern die Trennung von Natur und Geist ist selbst normativ, und das heißt für Pinkard, dass sie »gesetzt« und nicht einfach gegeben ist "The distinction between >mindedness and nature is itself something >posited ‘, that is, normative, not a metaphysical fact about ourselves that we discover ${ }^{20}{ }^{20}$

Laut Pinkard bedeutet Hegels berühmte Aussage in den Vorlesungen über die Philosophie des Geistes, derzufolge der Geist »sich zu dem macht, was er ursprünglich ist $^{21}$, dass Hegel hier eine »normative Konzeption« von Geist vertritt, die auf die Phänomenologie zurückweist. "Such a normative conception of smindedness had, of course, already been worked out in one direction in Hegel's own Phenomenology. As sminded, normative creatures we are, to use Charles Taylor's term, self-interpreting animals, not minds with bodies. The nature of mindedness had to do with how we took ourselves to be ${ }^{22}$

Zusammenfassend: Pinkard argumentiert, Hegel fasse den Unterschied zwischen Natur und Geist als ein Produkt des Geistes auf. Somit sei der Hegelsche Geist einer menschlichen, sozialen und normativen Instanz gleich. Dies impliziere, dass die ontologische Frage nach der Existenz und Seinsart des Geistes und der Natur im Verhältnis zum Geist von der Frage nach der Gesetzmäßigkeit der geistigen Tätigkeit ersetzt werden soll. Deswegen betont Pinkard mehrmals, dass die Differenz von Natur und Geist »normativ« und d. h.: »nicht-metaphysisch« sei. »Normativität« scheint darüber hinaus in Pinkards Sprachgebrauch synonym für »Allgemeinheit« und »Begrifflichkeit« zu sein. Durch die Bestimmung des Geistes als »normativ« will Pinkard mit Recht darauf aufmerksam machen, dass Hegel den Geist und den Unterschied zwischen Natur und Geist als Unterschied zwischen

16 Ebd.

17 Pinkard, German Philosophy, a. a. O., 277.

18 Ebd., 278.

19 Ebd.

20 Ebd., 279.

21 Hegel, Vorlesungen über die Philosophie des Geistes. Berlin 1827/1828, In: G. W. F. Hegel, Vorlesungen. Ausgewählte Schriften und Manuskripte, Bd. 13, hg. v. F. Hespe und B. Tuschling, Hamburg, 1994, 7.

22 Pinkard, German Philosophy, a. a. O., 279. 
Begriffen, Formen bzw. Funktionen und nicht als Differenz zwischen Substanzen oder Dingen konzipiert. Die Konzeption, dass »normativ« bzw. »prozessual« und »metaphysisch« sich gegenseitig ausschließen, scheint aber dabei problematisch.

Gegen die Entgegensetzung von »normativ« und »metaphysisch« kann man Verschiedenes einwenden. Eine ähnliche Entgegensetzung von Gesetzmäßigkeit, Funktionalität, Normativität und Metaphysik wurde im Neukantianismus entwickelt und ist aus verschiedenen Perspektiven hinterfragt worden. In der Tat kann die Normativität als eine Eigenschaft und Seinsart des Geistes aufgefasst werden, und daher als eine metaphysische Perspektive, die uns sagt, was der Geist ist. Dadurch, dass man behauptet, der Geist sei eine Funktion, stellen wir zugleich eine Metaphysik auf (wenn auch in Form einer Metaphysik der Funktionen, Normen und Gesetzen). Die Untrennbarkeit von Normativem und Deskriptiv-Metaphysischem scheint darüber hinaus der Grundzug der Kantischen und Hegelschen Umformulierung der metaphysischen Aufgabe. ${ }^{23}$

\section{Verteidigung der Metaphysik in der angloamerikanischen Hegeldeutung}

Polemisch gegen die antimetaphysische Interpretation Hegels ist eine andere Tendenz der angloamerikanischen Hegeldeutung, die sich insbesondere in den Arbeiten Frederick Beisers widerspiegelt. ${ }^{24}$ In The Puzzling Hegel Renaissance betont Beiser, Hegel antimetaphysisch zu interpretieren sei ein Zeichen historischer Ungenauigkeit. Er definiert die Metaphysik als »science of the absolute《 und das Absolute als »Self Positing Spirit «. ${ }^{25}$ Er beobachtet ferner, diese Ausdrücke seien aus einer heutigen Perspektive »seltsam« (»odd«) und diese Seltsamkeit stelle genau den Grund dar, warum die zeitgenössischen Interpreten versuchen, die Metaphysik (und das Absolute) aus der Hegelschen Philosophie zu beseitigen. Beiser schreibt: »The astonishing current Hegel-renaissance in Anglo American philosophy refuses metaphysics because contemporary culture mistrusts metaphysicsu. Deswegen: "Hegel scholarship is confronted with the following dilemma: if our scholarship is historically accurate, we confront a Hegel with profound metaphysical concerns, alien to the spirit of contemporary philosophical culture [...]. But if we continue interpreting Hegel in a nonmetaphysical manner, we have to accept that our interpretation is more a construction of our contemporary interests that the real historical school ${ }^{26}$

Eine ähnliche These über die Gründe der Ablehnung der Metaphysik im zeitgenössischen Neuhegelianismus verteidigt Pinkard. In What is the Non Metaphysical Reading of Hegel? betont er, dass das Vorhaben, Hegel nicht metaphysisch zu interpretieren dem Versuch entspricht, zu bestimmen, was von Hegel $»$ in an age of pragmatism, cognitive science and postmodernism is still alive «. ${ }^{27}$ D. h. die Kritik an der Metaphysik in der angloamerikanischen Hegeldeutung entspringe dem Versuch, Hegel aus einer zeitgenössischen Perspektive zu lesen.

23 Aus diesem Grund ist Strawsons Deutung der Kantischen Metaphysik als »descriptive metaphysiscs« ungeeignet, um die Normativität auszudrücken, die den Grundzug von Kants Auffassung der Kategorien, und somit seiner Neuformulierung der metaphysischen Aufgabe, ausmacht.

24 Frederick Beiser, »Hegel, A Non-Metaphysician? A Polemic Review of H. T. Engelhardt and T. Pinkard (eds), Hegel Reconsidered«, in: Bullettin of the Hegel Society of Great Britain, 32 (1995), 1-13. Ders., "Hegel and the Problem of Metaphysics«, in: The Cambridge Companion to Hegel, Cambridge, 1993, 1-24 und ders: »The Puzzling Hegel Renaissance«, in: The Cambridge Companion to Hegel, Cambridge, second and new edition, 2008.

25 Beiser, »Hegel and the Problem of Metaphysics«, a. a. O., 7.

26 Ebd.

27 Pinkard, »What ist the Non Metaphysical Reading of Hegel?«, a. a. O., 20. 
Im Gegenteil hierzu scheint sich jedoch die zeitgenössische Philosophie in eine andere Richtung zu entwickeln: die Sekundärliteratur zur Metaphysik und zu metaphysischen Themen ist entscheidend gewachsen, sodass man von einer Renaissance der Metaphysik auf sprachanalytischem Boden sprechen kann. So scheint es, dass Beisers Diagnose korrigiert werden sollte: eine metaphysische Hegeldeutung ist nicht nur wünschenswert, um den historischen Hegel wiederaufleben zu lassen, sondern auch, um seine Aktualität einzuschätzen.

Insgesamt scheinen Kritiker und Verteidiger der Metaphysik im angloamerikanischen Neuhegelianismus von einem restriktiven Begriff der Metaphysik auszugehen, der dem Hegelschen nicht entspricht. Sowohl die Kritiker als auch die Verteidiger teilen die traditionelle Idee der Metaphysik als "Wissenschaft vom Absoluten«, betonen die theologischen, mystischen und psychologischen Komponenten des Begriffs und vergessen dabei, dass die Metaphysik seit ihren Ursprüngen eng mit der Semantik verknüpft ist ${ }^{28}$ und dass es sowohl bei Kant als auch bei Hegel neben der Metaphysik, die kritisiert werden soll eine kritische und dialektische Metaphysik gibt, die verteidigt werden soll. $^{29}$

\section{Elena Ficara}

Technische Universität Berlin

Institut für Philosophie, Wissenschaftstheorie, Wissenschafts- und Technikgeschichte

Straße des 17. Juni 135

10623 Berlin

eficara@yahoo.de

28 Die Verknüpfung von Metaphysik und Begriffs- und Sprachanalyse wurde vor allem von Hans-Georg Gadamer (Wahrheit und Methode, Tübingen, 1960 und ders., Hegel Husserl Heidegger, Tübingen, 1987) und Ernst Tugendhat (Vorlesungen über die sprachanalytische Philosophie, Frankfurt/M., 1976) betont.

29 Diese These wird von verschiedenen zeitgenössischen deutschen Hegel-Interpreten betont, vgl. die Beiträge in dem Sammelband Metaphysik nach Kant?, hg. v. Dieter Henrich und Rolf-Peter Horstmann, Stuttgart, 1988; vgl. auch Klaus Düsing, Das Problem der Subjektivität in Hegels Logik. Systematische und entwicklungsgeschichtliche Untersuchungen zum Prinzip des Idealismus und zur Dialektik. Hegel Studien Beiheft 15. Bonn 1995; Rolf-Peter Horstmann, Ontologie und Relationen. Hegel, Bradley, Russell und die Kontroverse über interne und externe Beziehungen, Hain, 1984. Theunissen betont dagegen in Sein und Schein: die kritische Funktion der Hegelschen Logik, Frankfurt/M., 1978 die metaphysikkritische Funktion der Hegelschen Logik. 